\title{
Effect of superparamagnetic iron oxide nanoparticles on fluidity and phase transition of phosphatidylcholine liposomal membranes
}

This article was published in the following Dove Press journal:

International Journal of Nanomedicine

29 September 2015

Number of times this article has been viewed

\author{
Poornima Budime \\ Santhosh ${ }^{1, *}$ \\ Barbara Drašler ${ }^{2, *}$ \\ Damjana Drobne ${ }^{2}$ \\ Mateja Erdani Kreft ${ }^{3}$ \\ Slavko Kralj ${ }^{4}$ \\ Darko Makovec ${ }^{4}$ \\ Nataša Poklar Ulrih ${ }^{1,5}$ \\ 'Department of Food Science and \\ Technology, Biotechnical Faculty, \\ University of Ljubljana, ${ }^{2}$ Department \\ of Biology, Biotechnical Faculty, \\ University of Ljubljana, ${ }^{3}$ Institute of \\ Cell Biology, Faculty of Medicine, \\ University of Ljubljana, ${ }^{4}$ Department \\ for Materials Synthesis, Jožef Stefan \\ Institute, ${ }^{5}$ Centre of Excellence for \\ Integrated Approaches in Chemistry \\ and Biology of Proteins, Ljubljana, \\ Slovenia \\ *These authors share equal first \\ authorship
}

\begin{abstract}
Superparamagnetic iron oxide nanoparticles (SPIONs) with multifunctional properties have shown great promise in theranostics. The aim of our work was to compare the effects of SPIONs on the fluidity and phase transition of the liposomal membranes prepared with zwitterionic phosphatidylcholine lipids. In order to study if the surface modification of SPIONs has any influence on these membrane properties, we have used four types of differently functionalized SPIONs, such as: plain SPIONs (primary size was shown to be $\sim 11 \mathrm{~nm}$ ), silica-coated SPIONs, SPIONs coated with silica and functionalized with positively charged amino groups or negatively charged carboxyl groups (the primary size of all the surface-modified SPIONs was $\sim 20 \mathrm{~nm}$ ). Small unilamellar vesicles prepared with 1-palmitoyl-2-oleoyl-sn-glycero-3-phosphocholine lipids and multilamellar vesicles prepared with 1,2-dipalmitoyl-sn-glycero-3-phosphocholine lipids were encapsulated or incubated with the plain and surface-modified SPIONs to determine the fluidity and phase transition temperature of the bilayer lipids, respectively. Fluorescent anisotropy and differential scanning calorimetric measurements of the liposomes that were either encapsulated or incubated with the suspension of SPIONs did not show a significant difference in the lipid ordering and fluidity; though the encapsulated SPIONs showed a slightly increased effect on the fluidity of the model membranes in comparison with the incubated SPIONs. This indicates the low potential of the SPIONs to interact with the nontargeted cell membranes, which is a desirable factor for in vivo applications.
\end{abstract}

Keywords: encapsulated SPIONs, incubated SPIONs, zwitterionic liposomes, membrane integrity, phase behavior

\section{Introduction}

Liposomes encapsulating nanoparticles (NPs), bearing magnetic properties, called magnetoliposomes, have gained in popularity in the recent past owing to their potential applications especially in magnetic hyperthermia, ${ }^{1}$ targeted drug delivery ${ }^{2}$ and magnetic resonance imaging (MRI). ${ }^{3}$ Among the various magnetic NPs, superparamagnetic iron oxide nanoparticles (SPIONs) with improved surface properties are an excellent choice for in vivo applications due to their biocompatibility, nontoxicity, and are thus appropriate to be considered in nanotheranostics. ${ }^{4-8}$ These NPs with a small, singledomain core size $(<20 \mathrm{~nm})$ possess superparamagnetic properties and do not exhibit any spontaneous magnetic moments in the absence of an applied magnetic field. ${ }^{9,10}$ When the NPs approach a cell, they interact with the plasma membrane, which acts as a barrier to separate the internal contents of the cell from the external environment. ${ }^{11}$ A strong interaction of NPs with the liposomal membranes induces phase separation and fusion between the adjacent liposomes leading to a reduced stability of the vesicles. ${ }^{12-14}$ Since fluidity and phase behavior of the bilayer lipids influences various
Correspondence: Nataša Poklar Ulrih Department of Food Science and Technology, Biotechnical Faculty, University of Ljubljana, Jamnikarjeva I0I, 1000 Ljubljana, Slovenia

Tel +386 I 3230780

Fax +386 I 2566296

Email natasa.poklar@bf.uni-lj.si 
membrane-related properties as well as functions, it is vital to study the effect of different types of NPs and their mode of interactions with the model liposomal membranes.

To improve the stability of the SPIONs during in vivo applications, they are usually coated with different materials such as silica, polyethylene glycol, dextran, and citrate. ${ }^{15,16}$ Among the various coating materials, SPIONs coated with silica are widely used for bio-imaging and bio-sensing purposes due to their transparent matrix that allows the efficient passage of excitation and emission light through them. ${ }^{17,18}$ Hence, to explore the potential applications of silica coating and to compare the effects of silica-coated SPIONs with that of uncoated SPIONs, we have used plain SPIONs, silica-coated SPIONs, and surface-modified silicacoated SPIONs in the present work. For the efficient and safe in vivo applications of SPIONs, it is important that they do not alter the characteristics of the cell membranes. ${ }^{6,17-19}$ There are numerous literature data on the low reactivity of SPIONs against lipid membranes. ${ }^{20-22}$ Few reports have demonstrated that all types of SPIONs are not suitable for potential in vivo applications as some of them have a strong potential to alter the membrane properties based on different factors like surface charges, state of aggregation, and the nature of coating material. ${ }^{4,23}$ Therefore, it becomes essential to investigate the effect of SPIONs with different coating materials and surface charges on liposomes, prior to the in vivo applications.

Few studies have reported that the interaction of encapsulated and incubated NPs caused disturbances in the ordering of bilayer phospholipids, leading to an altered membrane structure and functions. ${ }^{24,25}$ But none of them have explained in detail the effect of SPIONs treated from the exterior and interior of the lipid bilayer. Hence, to get a clear understanding about the mechanisms of interactions between the SPIONs and model membranes, we have used two different methods. In the first method, the suspension of SPIONs was added during the hydration process of liposome formation, so that the SPIONs could be encapsulated inside the liposomes and has more probability to interact directly with the inner leaflet of the bilayer. In the second method, the SPIONs were incubated with the preformed vesicles so that presumably they interact more likely with the outer leaflet of the bilayer. In our previous works, we have investigated the effect of encapsulated SPIONs bearing different surface charges on the fluidity of zwitterionic liposomal membranes. ${ }^{26-28}$ In the present study, we would like to extend our work further by comparing the effects of plain- and surface-modified SPIONs treated from the inner and outer side of the liposomes. In addition to fluidity measurements, we have measured the phase transition profile of 1) liposomes encapsulated with the SPIONs and 2) liposomes incubated with the SPIONs to bring a correlation and to gain more knowledge about the changes induced in the thermodynamic state of the membrane lipids. For this purpose, we have measured the membrane fluidity of small unilamellar vesicles (SUVs) prepared from 1-palmitoyl-2-oleoyl-sn-glycero-3-phosphocholine (POPC) lipid (Figure 1A) and phase transition temperature of multilamellar vesicles (MLVs) prepared from 1,2-dipalmitoyl$s n$-glycero-3-phosphocholine (DPPC) lipid (Figure 1B). By using the very similar zwitterionic phospholipids POPC and DPPC, but with different phase transition characteristics, the effects of SPIONs on liposomal membranes existing in two different states, that is, at the liquid crystalline phase in POPC vesicles and gel crystalline phase in DPPC vesicles, were investigated. We have discussed that if the encapsulated SPIONs do not cause a significant alteration in the fluidity and phase transition temperature of the membrane lipids, then these NPs are suitable to be considered for encapsulation in liposomes for biomedical applications. Although the nature of artificial liposomes is far from the in vivo conditions with real biological membranes, phospholipid vesicles represent a widely used and a convenient model system for the assessment of interactions of different materials such as proteins, peptides or NPs with the membrane. ${ }^{7,12}$ If the incubation of SPIONs with the liposomes has a negligible effect on the above-mentioned properties, it is evident that their potential to interact with the nontargeted cell membranes is low, which is a desirable factor for in vivo applications such as magnetic separation, drug delivery, hyperthermia, and MRI contrast agents.

\section{Materials and methods}

The chemicals were reagent grade from commercial sources. Iron (III) sulfate hydrate, iron (II) sulfate heptahydrate (99+\%), citric acid (99+\%), tetraethoxysilane (99.9\%), 3-(2aminoethylamino)propylmethyldimethoxysilane (97\%), N,Ndimethylformamide (99\%), and succinic anhydride (99\%) were purchased from Alfa Aesar, Ward Hill, MA, USA. The acetone (AppliChem GmbH, Darmstadt, Germany), ethanol absolute (Carlo Erba, Val de Reuil, France, reagent - USP), $\mathrm{NH}_{4} \mathrm{OH}$ (aq) (Fluka, pa, 25\%), and $\mathrm{HCl} 1 \mathrm{M}$ (pa, Riedlde-Haën) were used as received. The lipids POPC and DPPC were purchased from Avanti Polar Lipids Inc., Alabaster, AL, USA. The fluorophores 1,6-diphenyl-1,3,5-hexatriene (DPH), (1-(4-trimethylammoniumphenyl)-6-phenyl-1,3,5hexatriene $p$-toluenesulfonate) (TMA-DPH) and other chemicals such as 4-(2-hydroxyethyl) piperazine-1-ethanesulfonic 

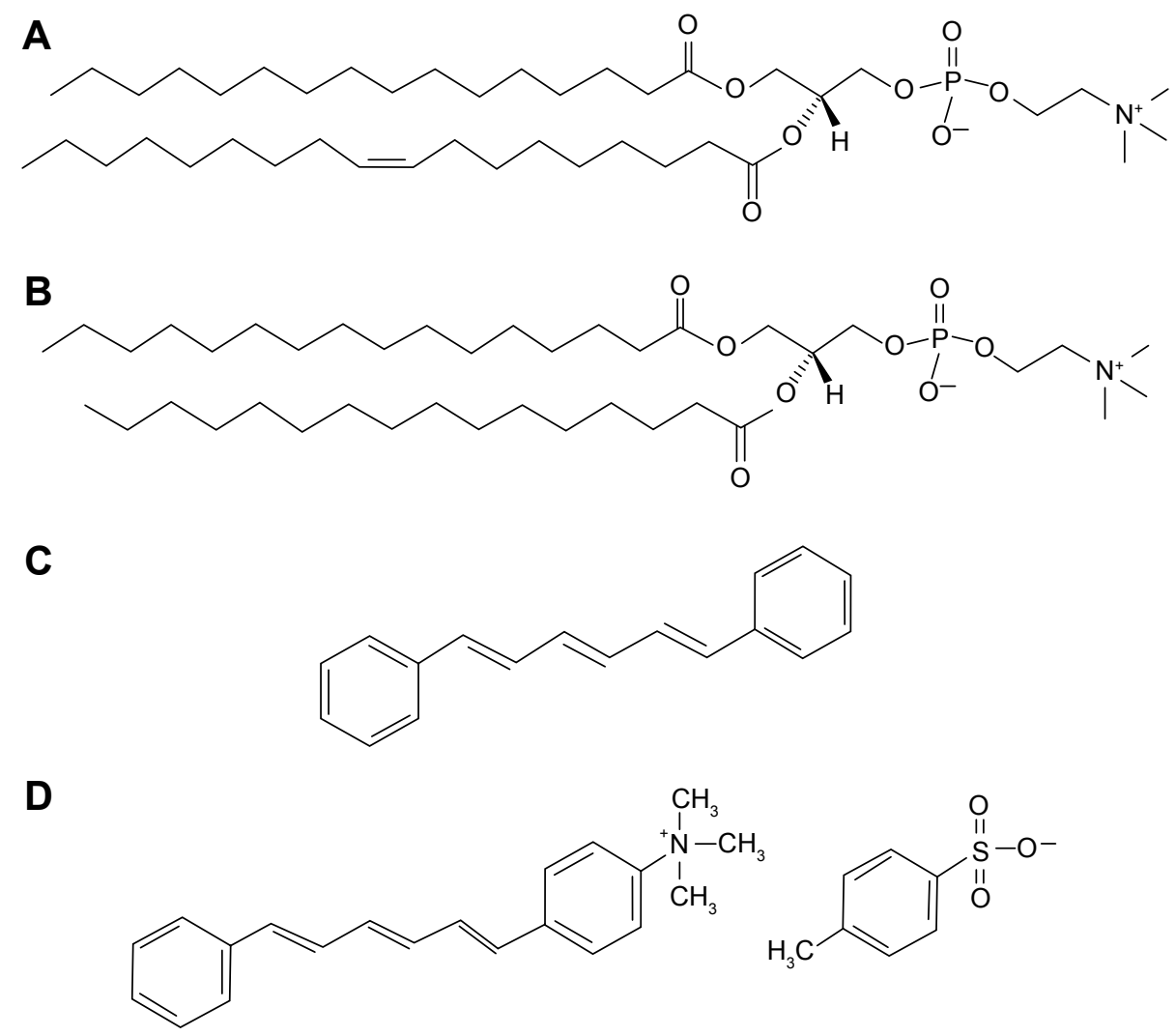

Figure I Structural formulas of the phospholipids and the fluorescent probes employed.

Notes: Structural formulas of (A) I-palmitoyl-2-oleoyl-sn-glycero-3-phosphocholine (POPC), (B) I,2-dipalmitoyl-sn-glycero-3-phosphocholine (DPPC), (C) I,6-diphenylI,3,5-hexatriene (DPH), and (D) (I-(4-trimethylammoniumphenyl)-6-phenyl-I,3,5-hexatriene p-toluenesulfonate) (TMA-DPH).

acid (HEPES), sepharose CL-4B, potassium thiocyanate $(\mathrm{KSCN})$, Triton X-100, chloroform and methanol were obtained from Sigma-Aldrich Chemie GmbH (Steinheim, Germany).

\section{Synthesis of surface modified SPIONs}

The superparamagnetic NPs of the iron oxide maghemite were synthesized using co-precipitation from aqueous solutions. Since the synthesized SPIONs show a strong tendency to agglomerate, they have to be dispersed in an aqueous medium prior to their coating with silica. The preparation of a stable aqueous suspension of NPs using citric acid as the surfactant has been described elsewhere. ${ }^{29}$ In brief, the synthesized SPIONs were incubated in a suspension containing citric acid, at $80^{\circ} \mathrm{C}$ for 90 minutes at a $\mathrm{pH}$ value of 5.2. After adsorption of the citric acid onto the SPIONs' surfaces, the $\mathrm{pH}$ value was raised to 10.2 using ammonia solution. After the excess citric acid was removed by ultrafiltration, the suspension was colloidally stable, that is, ferrofluid. Due to the charged carboxyl groups of citric acid, the NPs show strong negative surface charge, which electrostatically prevents the NPs from agglomeration.
The SPIONs in the stable aqueous suspension were further coated with an approximately $5 \mathrm{~nm}$ thick layer of silica by the hydrolysis and polycondensation of TEOS in the presence of an alkaline catalyst, as described elsewhere. ${ }^{30}$ In brief, the suspension of the citric acid-stabilized SPIONs was first diluted with ethanol (1:1 vol), and the $\mathrm{pH}$ was set to 11.0 using ammonia. Afterward, TEOS was added, and the mixture was magnetically stirred for 3 hours. The silica-coated SPIONs were then washed with ethanol twice using centrifugation $(14,000 \times g, 15$ minutes $)$. The formed silica heterogeneously nucleates and grows as a uniform layer on the surfaces of the SPIONs. To modify the surface charge of the silica-coated SPIONs, they were functionalized with amino and carboxyl groups. The amino-functionalized SPIONs were prepared by grafting 3-(2-aminoethylamino)propylmethyldimethoxysilane onto the surfaces of the silica-coated SPIONs. ${ }^{31}$ To prepare the carboxyl-functionalized SPIONs, the aminofunctionalized SPIONs were further functionalized with a ring-opening elongation reaction of the nonreacted surface amines with succinic anhydride providing the carboxyl functionalization. ${ }^{32}$ 


\section{Preparation of MLVs and SUVs}

MLVs and SUVs were prepared by thin film method using the rotary evaporator. ${ }^{33}$ Briefly, the SUVs were prepared by dissolving the POPC lipid in chloroform $(2 \mathrm{mg} / \mathrm{mL})$ in a round bottomed flask and argon gas was allowed to pass through the lipid-chloroform mixture to avoid the oxidation of lipids. The organic solvent was removed from the flask using the rotary evaporator (Buchirotavapor R-114; Büchi Labortechnik, Flawil, Switzerland) under very low pressure $(1.7 \mathrm{kPa})$, until a thin lipid film was formed at the bottom of the flask. The dried lipid film was then hydrated with $1 \mathrm{~mL}$ of $10 \mathrm{mM}$ HEPES buffer at $\mathrm{pH}$ 7.0. In order to encapsulate the SPIONs in liposomes, thin lipid films were prepared in a similar way and hydrated with the different suspensions of SPIONs containing $0.05 \mathrm{mg}$ of plain and surface-modified SPIONs $/ \mathrm{mL}$ in $10 \mathrm{mM}$ HEPES buffer (the ratio between the SPIONs and the phospholipids was assessed to be $\sim 2.4: 1$ $\left(\mathrm{n}\left(\mathrm{Fe}_{2} \mathrm{O}_{3}\right): \mathrm{n}(\mathrm{POPC})\right)$. A small amount of silanized glass beads was added to the flask and the sample was vortexed for 10 minutes to form MLVs. The obtained MLVs were then sonicated for 30 minutes total time with 10 seconds on-off cycles at $40 \%$ amplitude using a Vibracell Ultrasonic Disintegrator VCX 750 (Sonics and Materials Inc., Newtown, CT, USA) to form SUVs. The nonencapsulated SPIONs were separated from the sample by size exclusion chromatography (SEC) using sepharose gel (CL-4B column, $2.5 \times 1.5 \mathrm{~cm}$ ) and eluted with $10 \mathrm{mM}$ HEPES at $\mathrm{pH}$ 7.0. The separated liposomes were collected by a Retriever 500 fraction collector tube. To avoid the possibility of aggregated SPIONs with larger size to be co-eluted with the SUVs during the SEC method, the free SPIONs were treated by the same procedure used for liposome preparation without adding lipids and analyzed by SEC. The SUVs prepared by the above method were incubated individually with each type of SPIONs suspension $\left(0.05 \mathrm{mg} \mathrm{Fe} \mathrm{O}_{3} / \mathrm{mL}\right)$ for 50 minutes. The samples were then purified as mentioned above and used for analysis.

In addition to the SUVs prepared from POPC lipid, MLVs used in the differential scanning calorimetry (DSC) analysis were prepared from a stock solution of DPPC lipid in chloroform $(10 \mathrm{mg} / \mathrm{mL})$ in an identical way under the same conditions as for POPC MLVs, but with a final DPPC concentration of $0.5 \mathrm{mg} / \mathrm{mL}$. For both the encapsulation and incubation of DPPC MLVs with SPIONs, the same SPION dispersions were used following the same experimental procedures, but the final ratio between the SPIONs and

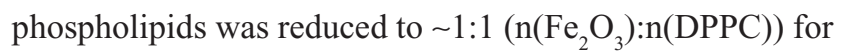
DSC analysis.

\section{Characterization of SPIONs and liposomes}

The SPIONs were characterized by transmission electron microscopy (TEM, JEOL 2100, JEOL, Tokyo, Japan) coupled with energy-dispersive X-ray spectroscopy (EDS). For TEM investigations, the SPIONs were deposited by drying the suspension of NPs on a copper grid-supported, perforated, transparent carbon foil. The NP size distribution was determined as an equivalent diameter from the TEM images using DigitalMicrograph $^{\mathrm{TM}}$ software. The statistical analyses of the NP size distributions were based on the measurements of the 300 SPIONs for each sample. The X-ray diffractometry (XRD) analysis of the precipitated SPIONs was performed using PANalytical X'Pert PRO diffractometer. The suspensions of SPIONs and SUVs were monitored with electro-kinetic measurements of the $\zeta$-potential (ZetaPALS, Brookhaven Instruments Corporation, Holtsville, NY, USA). The hydrodynamic size distribution of the SUVs and SPIONs in their aqueous suspensions was obtained using dynamic light scattering (DLS) (ANALYSETTE 12 DynaSizer, Fritsch, Oberstein, Germany).

In order to visualize the magnetoliposomes using the TEM analysis, control DPPC MLVs and DPPC MLVs encapsulated with silica-coated SPIONs were prepared following the same procedure as described above. To avoid the excess of SPIONs in TEM samples, the molar ratio of DPPC: $\mathrm{Fe}_{2} \mathrm{O}_{3}$ was $4: 1$. A droplet $(5 \mu \mathrm{L})$ containing diluted suspensions of MLVs of each specimen (1:100 in $10 \mathrm{mM}$ HEPES buffer) was spread onto the formvar/carbon-coated grids (UV-treated, 5 minutes). The samples were allowed to dry (during the night, room temperature) and then a droplet $(5 \mu \mathrm{L})$ of freshly prepared $1 \%$ ammonium molybdate solution in $\mathrm{dH}_{2} \mathrm{O}(\mathrm{pH}$ 4.7) was spread over the samples for 2 minutes, washed three times in $\mathrm{dH}_{2} \mathrm{O}$ and left until dried (following the procedure above, the DPPC lipids were in the fully hydrated state).

\section{Assay of phospholipids}

The concentration of phospholipids in SUVs and MLVs was determined by a spectrophotometric test based on the enzymatic reaction, as described by the manufacturer (Wako Phospholipids C Assay, Wako Pure Chemical Industries, Richmond, Japan). In brief, the color reagent was prepared by dissolving the chromogen substrate in Good's buffer ( $\mathrm{pH}$ 7.5). To this, an appropriate amount of the standard phospholipid solution was added to achieve the final phospholipids' concentrations such as $150 \mathrm{mg} / \mathrm{dL}, 300 \mathrm{mg} / \mathrm{dL}$ and $596.1 \mathrm{mg} / \mathrm{dL}$, respectively. Approximately $10 \mu \mathrm{L}$ of the sample liposomes were treated with $1.5 \mathrm{~mL}$ of buffer containing the color reagent and incubated at $37^{\circ} \mathrm{C}$ for 5 minutes. The enzymes present in 
the color reagent react with the phospholipids to produce a blue-colored product, the absorbance of which was measured at $600 \mathrm{~nm}$ and $700 \mathrm{~nm}$, using Hewlett Packard 8453E UV-vis spectrophotometer (Hewlett-Packard, Waldbronn, Germany). A calibration curve was plotted to measure the final lipid concentration by comparing the known values of standard phospholipids in the kit under the same conditions.

\section{Encapsulation efficiency of magnetoliposomes}

The encapsulation efficiency ( $\mathrm{EE} \%$ ) of liposomes denotes the amount of the cargo that is loaded inside the vesicles. The $\mathrm{EE} \%$ was determined by measuring the ratio of iron per mole of the phospholipid present in the liposomes before and after the purification process. To check if the surface charge of SPIONs has any influence on the encapsulation yield, we have measured the $\mathrm{EE} \%$ of magnetoliposomes encapsulated with negatively charged silica-coated SPIONs and positively charged SPIONs functionalized with amino groups. The iron content in magnetoliposomes was calculated using a colorimetric method. ${ }^{4,34}$ In brief, approximately $60 \mu \mathrm{L}$ of the SEC purified and nonpurified liposome suspensions were treated with $15 \mu \mathrm{L}$ of the surfactant TritonX-100 (1\% [v/v] in final solution) to disrupt the vesicles and release the encapsulated SPIONs. To this mixture, $0.675 \mathrm{~mL}$ of $37 \%$ $\mathrm{HCl}$ and $0.750 \mathrm{~mL}$ of $50 \mathrm{mM} \mathrm{KSCN}$ color reagent was added. The sample was vortexed, and the absorbance was read at $480 \mathrm{~nm}$ (Hewlett Packard 8453E UV-vis spectrophotometer, Hewlett-Packard). A calibration curve was plotted with aqueous suspensions of both types of SPIONs in the presence and absence of surfactants and lipids to determine the accuracy of this method for magnetoliposomes.

\section{Fluorescence anisotropy measurements}

The effect of SPIONs on the membrane fluidity of POPC SUVs was investigated by the fluorometric method using the fluorescent probes, DPH (Figure 1C) and its cationic derivative TMA-DPH (Figure 1D). To study the effect of SPIONs on membrane fluidity, we have prepared SUVs encapsulated with SPIONs and SUVs incubated with SPIONs for 50 minutes. Temperature-dependent fluorescence anisotropy measurements of the probes incorporated in the liposomal membranes were performed in 10-mm-path-length cuvettes using a Cary Eclipse fluorescence spectrophotometer (Varian, Mulgrave, Australia). In the cuvettes, either $10 \mu \mathrm{L}$ of DPH or TMA-DPH was added to $2.5 \mathrm{~mL} 100 \mu \mathrm{M}$ solutions of SUVs to obtain a final concentration of $0.5 \mu \mathrm{M}$ DPH and $1.0 \mu \mathrm{M}$ TMA-DPH. The anisotropy values of SUVs were measured within the temperature ranging from $15^{\circ} \mathrm{C}$ to $50^{\circ} \mathrm{C}$, by increasing the temperature by $5^{\circ} \mathrm{C}$ for every measurement, with a time interval of 15 minutes with constant mixing. Varian autopolarizers with slit widths with a nominal band-pass of $5 \mathrm{~nm}$ were used for both excitation and emission. DPH and TMADPH fluorescence anisotropy values were measured at the excitation wavelength of $358 \mathrm{~nm}$ and emission wavelength of $410 \mathrm{~nm}$. The anisotropy $\langle r\rangle$ values were calculated as shown in Equation 1 using the built-in software of the instrument:

$$
\langle r\rangle=\frac{I_{\|}-G I_{\perp}}{I_{\|}+2 G I_{\perp}}
$$

where, $I_{\|}$and $I_{\perp}$ are the emission intensities with polarizers parallel and perpendicular to the direction of the polarized exciting light, respectively. The values of the G-factor (ratio of the sensitivities of the detection system for vertically [IHV] and horizontally $[\mathrm{IHH}]$ polarized light) were determined separately for each sample. Applying Equation 2, the lipidorder parameter $S$ values were calculated from the anisotropy values using the following expression: ${ }^{35}$

$$
S=\frac{\left[1-2\left(\frac{r}{r_{0}}\right)+5\left(\frac{r}{r_{0}}\right)^{2}\right]^{\frac{1}{2}}-1+\frac{r}{r_{0}}}{2\left(\frac{r}{r_{0}}\right)}
$$

where $r_{0}$ is the fluorescence anisotropy of DPH in the absence of any rotational motion of the probe. The order parameter values of the samples were compared with the control SUVs without SPIONs to study the effect of SPIONs from inside and outside of the membrane.

\section{Differential scanning calorimetry}

The DSC is a very sensitive and convenient technique for monitoring phase transition behavior of lipid membranes and allows the detection of even minor changes in the state of the membrane lipids. ${ }^{36,37}$ Since DPPC is a widely used long chain phospholipid with a well-characterized gel to liquid phase transition state $\left(T_{m} \sim 41^{\circ} \mathrm{C}\right)$, we have used them to study the alterations in the phase transition temperature using a Nano DS series III calorimeter (Calorimetry Science, Provo, UT, USA). The samples loaded into the calorimetric cell were heated and cooled repeatedly in the temperature ranging from $10^{\circ} \mathrm{C}$ to $70^{\circ} \mathrm{C}$, with the heating rate of $1^{\circ} \mathrm{C} \mathrm{min}^{-1}$. The first DSC scan was used to determine the temperature of the gel-to-liquid crystalline phase transition temperature $\left(T_{m}\right)$ of the lipids and 
the calorimetric enthalpy $\left(\Delta H_{c a l}\right)$ as explained previously. ${ }^{38}$ The subsequent scans were used to determine the reversibility of the lipid-phase transitions. The OriginPro 8.1 software (OriginLab Corporation, Northampton, MA, USA) was used to evaluate the enthalpies and transition temperatures of the lipids from the DSC thermograms.

\section{Results}

\section{Characteristics of SPIONs, liposomes, and lipid assay}

The particle size of the plain SPIONs measured from TEM images was found to be $11.6 \pm 2.7 \mathrm{~nm}$ (Figure 2A). The amorphous layer of silica appears to be homogeneous with a fairly constant thickness, close to $5 \mathrm{~nm}$ (Figure 2B-D). XRD of the plain SPIONs showed a single spinel phase (Figure 2E), whereas the chemical analyses showed that there was less than $3 \%$ of iron present in the oxidation state $2+$, confirming that the NPs were composed of maghemite. ${ }^{30}$

The changes in the surface charge of the SPIONs were followed by the zeta $(\zeta)$ potential measurements of their aqueous suspensions (pH 7.4) and in $10 \mathrm{mM} \mathrm{HEPES} \mathrm{buf-}$ fer ( $\mathrm{pH}$ 7.0). The $\zeta$-potential values of the plain SPIONs, silica-coated SPIONs, SPIONs functionalized with amino or carboxyl groups are presented in Figure 2F. The suspensions
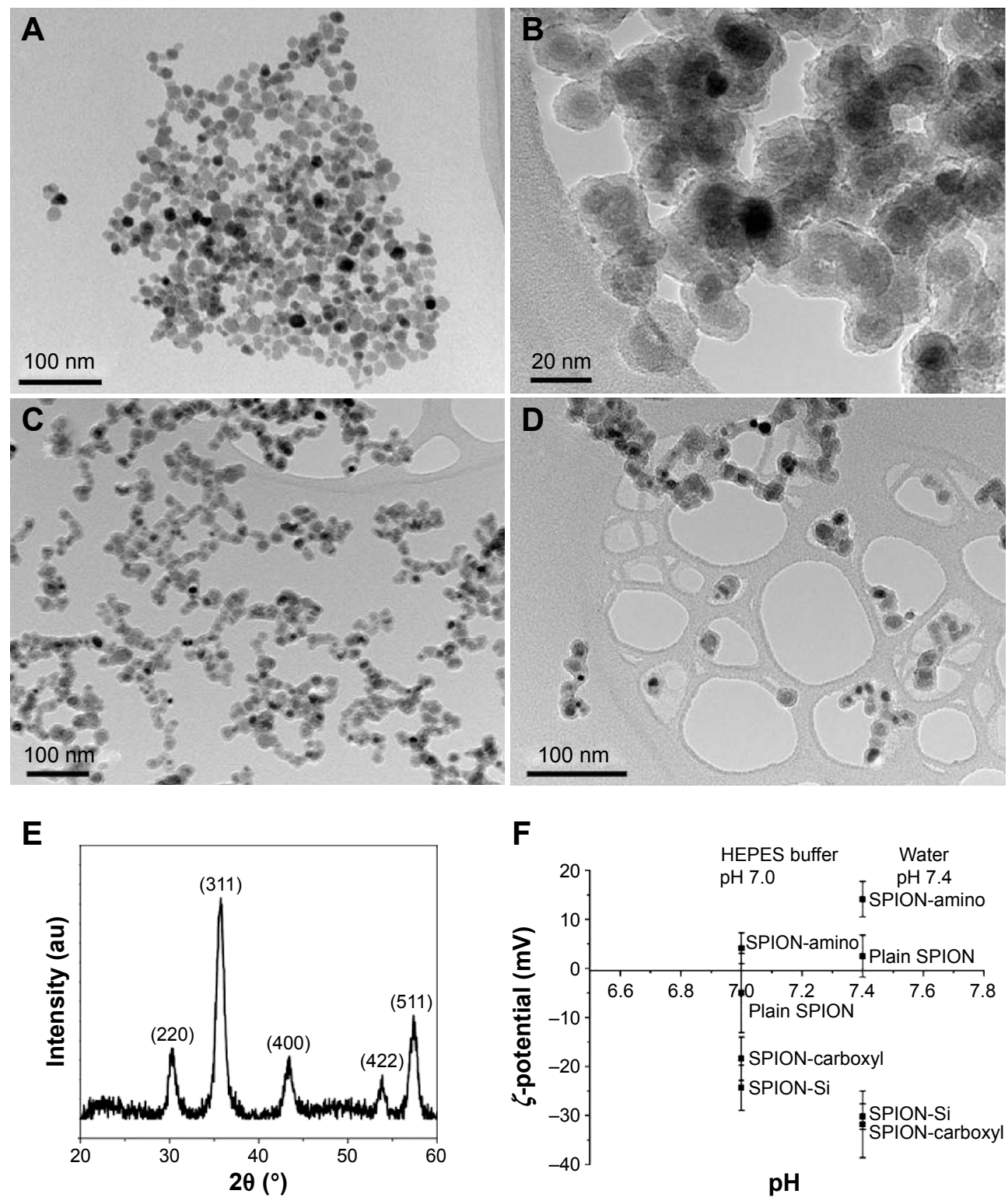

Figure 2 Characteristics of SPIONs and SPION suspensions.

Notes: TEM images of (A) plain SPIONs, (B) silica-coated SPIONs, (C) SPIONs functionalized with amino groups, (D) SPIONs functionalized with carboxyl groups, (E) the XRD pattern of the plain SPIONs, and (F) $\zeta$-potential measurements of all types of SPIONs in water and in $10 \mathrm{mM} \mathrm{HEPES} \mathrm{buffer} \mathrm{(pH} \mathrm{7.0).}$

Abbreviations: TEM, transmission electron microscopy; HEPES, 4-(2-hydroxyethyl) piperazine-I-ethanesulfonic acid; SPIONs, superparamagnetic iron oxide nanoparticles; $\mathrm{XRD}, \mathrm{X}$-ray diffractometry. 
of the plain SPIONs showed a relatively low absolute value of the $\zeta$-potential close to neutral $\mathrm{pH}$, since the isoelectric point (IEP) of the as-synthesized SPIONs $\mathrm{pH}$ value was approximately $7.0 .{ }^{31} \mathrm{~A}$ low absolute value of the $\zeta$-potential usually correlates with the poor colloidal stability of such suspensions. The silica-coated SPIONs show a relatively acidic character, because its structure is terminated with negatively charged hydroxyl groups for the whole range of considered $\mathrm{pH}$ values. The amino functionalization provides the positive surface charge at neutral $\mathrm{pH}$ values due to the shift of the IEP to a higher $\mathrm{pH}$ value of approximately 8.0. Due to the acidic character of the surface carboxyl groups, the carboxyl functionalization displays the IEP at a $\mathrm{pH}$ value of approximately 4.0 providing negative $\zeta$-potential at the neutral $\mathrm{pH}$ values. ${ }^{32}$ The presence of salts in the HEPES buffer solution influences the $\zeta$-potential of SPIONs and consecutively alters the colloidal stability of the suspensions. Usually, the presence of salts lowers the absolute value of the $\zeta$-potential due to the screening of the colloidal surface charge and thus makes the electrostatic repulsions less pronounced, which normally results in an impaired colloidal stability.

The average hydrodynamic sizes of SPIONs in their aqueous suspensions and in the experimental buffer $(10 \mathrm{mM}$ HEPES) were measured using DLS and are listed in Table 1. The plain SPIONs without coating formed agglomerates, whereas silica - coated and carboxyl group-functionalized SPIONs were well dispersed. Though amino-functionalized SPIONs formed some agglomerates in HEPES buffer, they did not sediment in the suspension as fast as the plain SPIONs. The hydrodynamic size measured before any visible sedimentation was significantly larger for the SPIONs in the HEPES buffer compared to the SPIONs in water. The increase in the hydrodynamic size is the consequence of

Table I Characterization data of the plain and surface modified SPIONs

\begin{tabular}{|c|c|c|c|}
\hline Sample & $\begin{array}{l}\text { Primary } \\
\text { particle } \\
\text { size }(\mathrm{nm}) ; \\
\text { diameter } \pm \text { SD }\end{array}$ & $\begin{array}{l}\text { Hydrodynamic } \\
\text { size in water } \\
(\mathrm{nm}) \text {; PDI }\end{array}$ & $\begin{array}{l}\text { Hydrodynamic } \\
\text { size in } 10 \mathrm{mM} \\
\text { HEPES (nm); } \\
\text { PDI }\end{array}$ \\
\hline Plain SPIONs & $11.6 \pm 2.7$ & $\mathrm{a}$ & a \\
\hline $\begin{array}{l}\text { Silica-coated } \\
\text { SPIONs }\end{array}$ & $20.7 \pm 4.2$ & $26 \mathrm{~nm} ; 0.12$ & $30 \mathrm{~nm} ; 0.14$ \\
\hline $\begin{array}{l}\text { Carboxyl groups } \\
\text { functionalized } \\
\text { SPIONs }\end{array}$ & $20.7 \pm 4.2$ & $38 \mathrm{~nm} ; 0.18$ & $33 \mathrm{~nm} ; 0.18$ \\
\hline $\begin{array}{l}\text { Amino groups } \\
\text { functionalized } \\
\text { SPIONs }\end{array}$ & $20.7 \pm 4.2$ & $47 \mathrm{~nm} ; 0.22$ & 173 nm; 0.48 \\
\hline
\end{tabular}

Note: ${ }^{2}$ The result was not reliable due to intensive agglomeration.

Abbreviations: PDI, polydispersity index; HEPES, 4-(2-hydroxyethyl) piperazine- Iethanesulfonic acid; SPIONs, superparamagnetic iron oxide nanoparticles. the lower absolute value of their $\zeta$-potential in the HEPES buffer due to increased salinity and increased buffer $\mathrm{pH}$ value ( $\mathrm{pH}$ 7.0) that is close to the IEP of the amino-functionalized SPIONs. In contrast, the presence of the HEPES buffer did not significantly affect the hydrodynamic sizes of both suspensions containing the silica-coated SPIONs and the carboxyl-functionalized SPIONs, despite of decreased absolute values of their $\zeta$-potential. The IEPs of both suspensions were at much lower $\mathrm{pH}$ values and thus even the presence of salts did not significantly impair the colloidal stability. ${ }^{32}$

The TEM micrographs of DPPC MLVs encapsulated with silica-coated SPIONs in $10 \mathrm{mM}$ HEPES buffer ( $\mathrm{pH} 7.0$ ) were depicted in Figure 3A. The images show the interaction of SPIONs with the membranes of DPPC MLVs. Further, the colocalization of SPIONs (encapsulated dark material) and the organic material was confirmed using energy EDS (Figure 3B). The EDS analysis, capturing the whole region (Figure 3A), confirmed the presence of SPIONs (black material) and that the surrounding gray area corresponds to the organic matter/phospholipids. Apart from the elements, such as phosphorous, carbon, nitrogen, and oxygen, which indicate the presence of organic material, the EDS spectra has also shown the peaks corresponding to molybdenum in the contrasting agent and to both the elements $\mathrm{Fe}$ and $\mathrm{Si}$ which confirms the presence of silica-coated SPIONs in the liposomes.

The size range of the sonicated control SUVs without SPIONs and SUVs encapsulated with SPIONs was found to be between $60 \mathrm{~nm}$ and $150 \mathrm{~nm}$ using DLS, with a polydispersity index of $0.10 \pm 0.01$. The initial lipid concentration used to prepare the SUVs was $2 \mathrm{mg} / \mathrm{mL}$. The phospholipid assay results revealed a final lipid concentration of SUVs to be $1.8 \mathrm{mg} / \mathrm{mL}$, which indicates that there was not much lipid loss during the entire procedure. Based on this value, the lipid concentration was adjusted for all other measurements.

The $\zeta$-potential value of control SUVs without SPIONs was found to be $-4.0 \pm 0.4 \mathrm{mV}$ and the values for SUVs encapsulated with plain, silica-coated SPIONs and those functionalized with amino and carboxyl groups were found to be $-6.7 \pm 3.4 \mathrm{mV},-7.2 \pm 3.0 \mathrm{mV},-6.9 \pm 1.8 \mathrm{mV}$, and $-10.7 \pm 1.9 \mathrm{mV}$, respectively. The results have depicted that the SPIONs decreased the $\zeta$-potential values of magnetoliposomes probably due to their interaction with the bilayer lipid chains. The amino functionalized SPIONs decreased the $\zeta$-potential values of magnetoliposomes comparably to the negatively charged silica-coated SPIONs and those SPIONs functionalized with carboxyl groups. But they did not cause positive $\zeta$-potential values of magnetoliposomes, presumably 


\section{A}

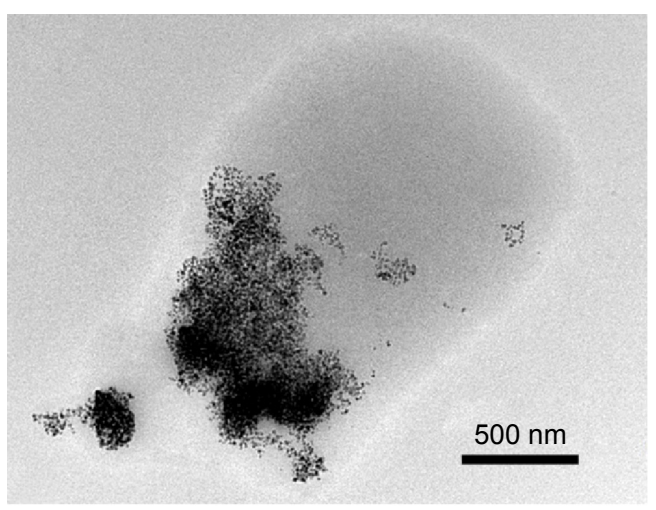

B

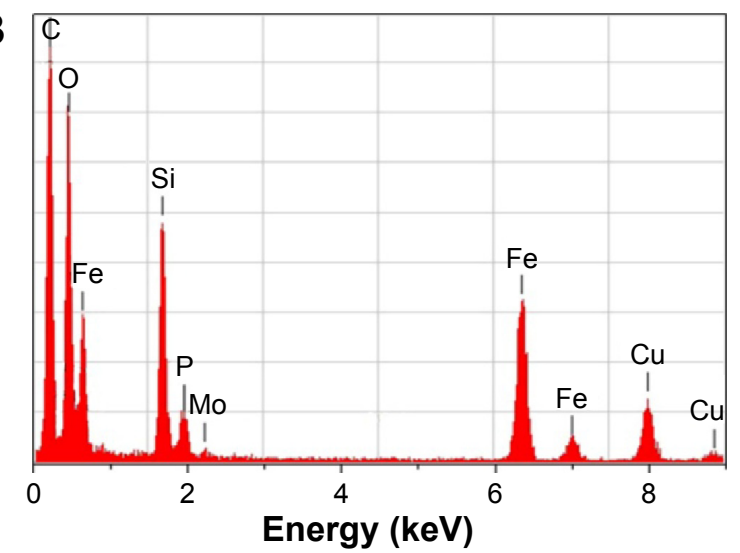

Figure 3 TEM micrograph and the corresponding elementary composition of DPPC multilamellar vesicles, encapsulated with silica-coated SPIONs.

Notes: (A) TEM micrograph of DPPC MLVs with silica-coated SPIONs. The scale bar is $500 \mathrm{~nm}$. (B) Elementary composition of the selected area, including both silica-coated SPIONs and adjusting organic material (amorphous material). Both elements Fe and Si confirm presence of silica-coated SPIONs (remark: presence of Si is characteristic also for the background signal). Observed elements $\mathrm{P}, \mathrm{C}, \mathrm{N}$, and $\mathrm{O}$ confirm presence of organic material (phospholipids, though $\mathrm{C}$ is also spread on the Cu-grids). Observed the presence of $\mathrm{Mo}$ is a consequence of ammonium molybdate used as a contrast agent.

Abbreviations: TEM, transmission electron microscopy; DPPC, I,2-dipalmitoyl-sn-glycero-3-phosphocholine; MLVs, multilamellar vesicles; SPIONs, superparamagnetic iron oxide nanoparticles; POPC, I-palmitoyl-2-oleoyl-sn-glycero-3-phosphocholine.

due to their very low positive surface charge $(4.1 \pm 3.1 \mathrm{mV})$ in the relevant medium (HEPES buffer). Another reason could be that the inclusion of positively charged iron oxide NPs decreased the $\zeta$-potential values of zwitterionic liposomes and the association of NPs caused a decrease in the exposure of positive charges of the lipid headgroups, possibly due to the reorganization of bilayer lipids as a result of NPs interaction with the membrane as demonstrated by Philosof-Mazor et al. ${ }^{39}$

\section{Encapsulation efficiency}

The iron content in the magnetoliposomes was determined spectrophotometrically using the KSCN reagent, and the $\mathrm{EE} \%$ was measured by calculating the ratio between iron and lipid in magnetoliposomes before and after SEC purification. The EE\% of magnetoliposomes loaded with silica-coated SPIONs and SPIONs functionalized with amino groups is shown in Table 2. The results have shown a variation in EE\% corresponding to the initial proportion of SPIONs encapsulated in SUVs. Before purification, the quantity of iron in the magnetoliposomes suspension was high that was reduced comparatively after purification by the efficient removal of the nonencapsulated SPIONs using SEC.

The highest encapsulation percentage of $78 \%$ was achieved for silica-coated SPIONs loaded magnetoliposomes with the lowest initial iron content of $0.01 \mathrm{mg} \mathrm{Fe} / \mathrm{mL}$, whereas the lowest encapsulation percentage of $54 \%$ was achieved for the SPIONs functionalized with amino groups

Table 2 Encapsulation efficiency of MLs loaded with surface modified SPIONs

\begin{tabular}{|c|c|c|c|c|c|c|}
\hline \multirow{2}{*}{$\begin{array}{l}\text { Initial iron } \\
\text { concentration } \\
\text { in MLs } \\
(\mathrm{mg} \mathrm{Fe} / \mathrm{mL})\end{array}$} & \multicolumn{2}{|c|}{$\begin{array}{l}\text { Concentration of iron in MLs } \\
\text { before purification } \\
\text { (g Fe/mol POPC) }\end{array}$} & \multicolumn{2}{|c|}{$\begin{array}{l}\text { Concentration of iron in MLs } \\
\text { after purification } \\
\text { (g Fe/mol POPC) }\end{array}$} & \multicolumn{2}{|l|}{ Percentage of EE\% } \\
\hline & $\begin{array}{l}\text { MLs loaded with } \\
\text { silica-coated } \\
\text { SPIONs }\end{array}$ & $\begin{array}{l}\text { MLs loaded with } \\
\text { amino groups } \\
\text { functionalized } \\
\text { SPIONs }\end{array}$ & $\begin{array}{l}\text { MLs loaded with } \\
\text { silica-coated } \\
\text { SPIONs }\end{array}$ & $\begin{array}{l}\text { MLs loaded with } \\
\text { amino groups } \\
\text { functionalized } \\
\text { SPIONs }\end{array}$ & $\begin{array}{l}\text { MLs loaded with } \\
\text { silica-coated } \\
\text { SPIONs }\end{array}$ & $\begin{array}{l}\text { MLs loaded with } \\
\text { amino groups } \\
\text { functionalized } \\
\text { SPIONs }\end{array}$ \\
\hline 0.01 & $51.4 \pm 2.1$ & $43.1 \pm 1.8$ & $40.1 \pm 2.9$ & $30.7 \pm 2.3$ & $78 \pm 6.5$ & $7 I \pm 6 . I$ \\
\hline 0.05 & $57.1 \pm 3.4$ & $54.2 \pm 1.7$ & $42.3 \pm 3.9$ & $35.3 \pm 2.4$ & $74 \pm 8.1$ & $65 \pm 4.9$ \\
\hline 0.2 & $67.8 \pm 4.1$ & $67.8 \pm 2.3$ & $46.8 \pm 5.2$ & $41.4 \pm 3.7$ & $69 \pm 8.7$ & $61 \pm 5.8$ \\
\hline 0.5 & $80.3 \pm 6.5$ & $81.6 \pm 3.1$ & $52.2 \pm 3.2$ & $48.2 \pm 3.6$ & $65 \pm 6.6$ & $59 \pm 4.9$ \\
\hline 1.0 & $162.1 \pm 0.1$ & $167.5 \pm 2.3$ & $98.9 \pm 1.3$ & $95.5 \pm 0.3$ & $61 \pm 7.9$ & $57 \pm 7.4$ \\
\hline 2.0 & $330.5 \pm 2.8$ & $339.2 \pm 4.7$ & $188.4 \pm 4.3$ & $183.2 \pm 5.7$ & $57 \pm 4.9$ & $54 \pm 5.2$ \\
\hline
\end{tabular}

Notes: Encapsulation efficiency of MLs loaded with SPIONs functionalized with amino groups and silica-coated SPIONs was determined from the percentage of iron content in magnetoliposomes before and after the purification process. EE\% values $( \pm S D)$ are the mean of five calculations.

Abbreviations: MLs, magnetoliposomes; POPC, I-palmitoyl-2-oleoyl-sn-glycero-3-phosphocholine; EE\%, encapsulation efficiency; SPIONs, superparamagnetic iron oxide nanoparticles. 
loaded magnetoliposomes with the highest initial iron content of $2.0 \mathrm{mg} \mathrm{Fe} / \mathrm{mL}$. It has been reported that the $\mathrm{Fe}^{2+}$ and $\mathrm{Fe}^{3+}$ ions have a strong affinity to bind with the negatively charged phosphate groups of the membrane lipids and their subsequent encapsulation in the vesicles. ${ }^{27,40,41}$ Increasing the initial iron concentration further up to $5 \mathrm{mg} / \mathrm{mL}$ did not show a considerable increase in the encapsulation of SPIONs in magnetoliposomes (data not shown) which is in line with other reports. ${ }^{4,42}$

\section{Effects of SPIONS on membrane fluidity}

The comparison of the order parameter $(S)$ values of POPC SUVs encapsulated and incubated with all four types of SPIONs is shown in Figure 4A-D. As the temperature increased, a gradual decrease in the order of parameter values of the bilayer lipids was observed for the SUVs encapsulated and incubated with all the types of SPIONs using the probes DPH and TMA-DPH. In comparison with the control, a little increase in the $S$ value was noted for the SUVs encapsulated with all types of tested SPIONs using DPH and TMA-DPH (Figure 4A and B). The initial $S$ value for control SUVs at $15^{\circ} \mathrm{C}$ was found to be $0.51 \pm 0.04$. The highest $S$ value of $0.65 \pm 0.03$ and $0.75 \pm 0.01$ were observed using DPH and TMA-DPH for the SUVs encapsulated with SPIONs functionalized with amino groups, respectively, at the same temperature. Among the SUVs incubated with the SPIONs (Figure 4C and D), the highest $S$ value of $0.63 \pm 0.03$ and $0.74 \pm 0.01$ was recorded at $15^{\circ} \mathrm{C}$ using
A
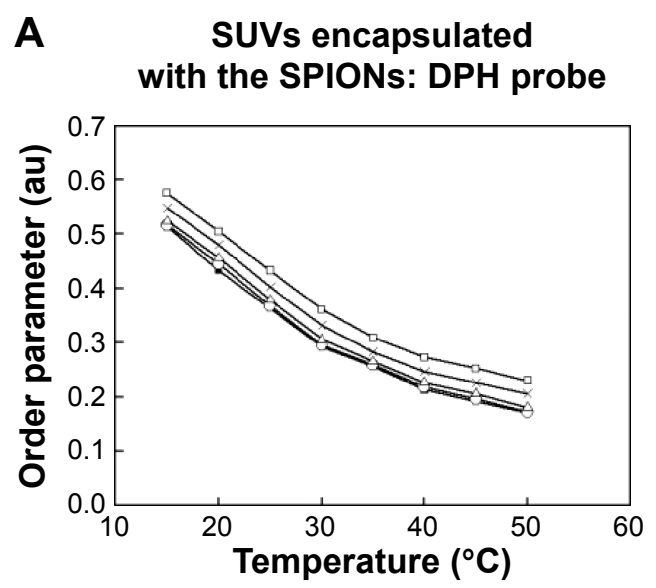

C

C SUVs incubated with the SPIONs: DPH probe

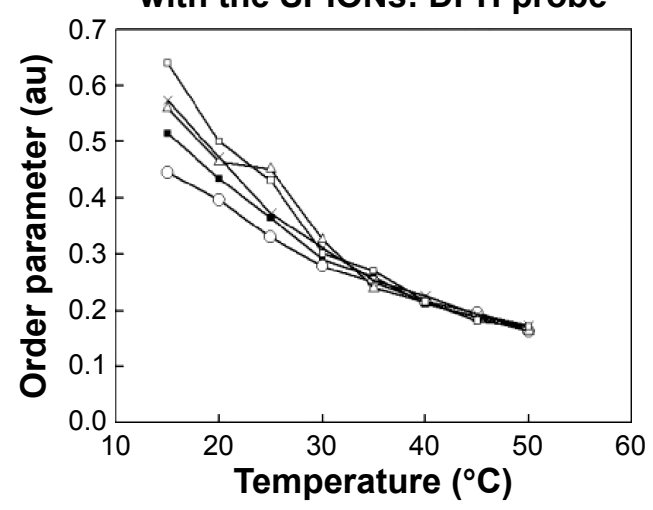

B
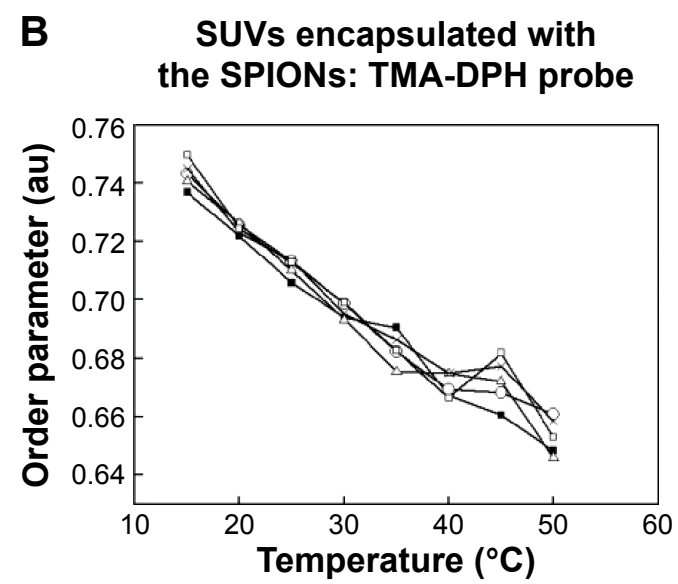

D SUVs incubated with

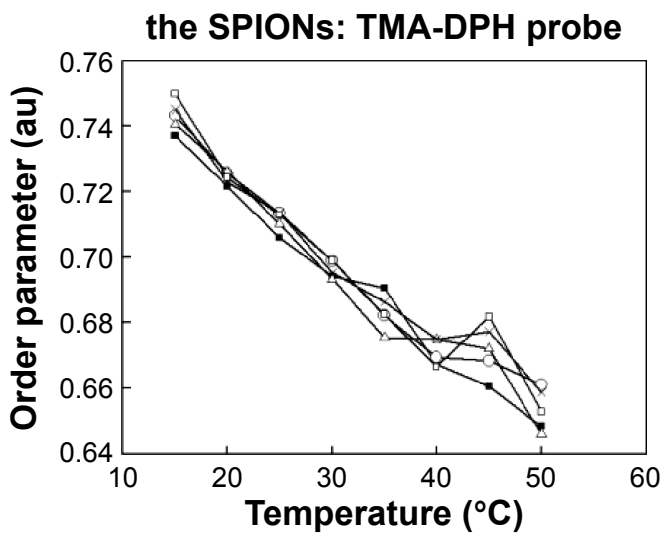

$\rightarrow$ Control $\quad \rightarrow$ - Plain SPIONs $\leadsto$ Silica coated SPIONs

$*$ Carboxyl group functionalized SPIONs $\quad \rightarrow$ - Amino group functionalized SPIONs

\footnotetext{
Figure 4 Comparison of the order parameter of the POPC SUVs either encapsulated or incubated with the suspensions of the SPIONs.

Notes: Panels (A and B) show the order parameter values of SUVs encapsulated with the SPIONs, assessed using the probes (A) DPH, and (B) TMA-DPH. Panels (C and D) show the order parameter of SUVs incubated with SPIONs, using (C) DPH and (D) TMA-DPH (- - - Control SUVs without SPIONs; $-\bigcirc-$ SUVs with plain SPIONs; - - - SUVs with silica-coated SPIONs; -X-SUVs with carboxyl groups functionalized SPIONs; $-\square-$ SUVs with amino groups functionalized SPIONs). The order parameter values are the mean of two measurements. The error value denotes the standard errors $( \pm S D)$ of sample mean values during the analysis.

Abbreviations: SD, standard deviation; SUV, small unilamellar vesicle; SPIONs, superparamagnetic iron oxide nanoparticles; DPH, I,6-diphenyl-I,3,5-hexatriene; TMA-DPH (I-(4-trimethylammoniumphenyl)-6-phenyl-I,3,5-hexatriene p-toluenesulfonate).
} 
DPH and TMA-DPH for the SPIONs functionalized with amino groups, respectively. However, in this case, higher discrepancies in $S$ values were observed at lower temperatures using DPH probe and at higher temperatures using TMA-DPH probe. This suggests that the interaction of the tested SPIONs was higher at the initial stages of incubation and at higher temperatures where the fluidity of the membrane was high. In comparison with the control, the order parameter values for both the encapsulated and incubated SUVs increased in the order of plain, silica-coated SPIONS, SPIONs functionalized with carboxyl groups, and SPIONs functionalized with amino groups using both the probes (DPH and TMA-DPH).
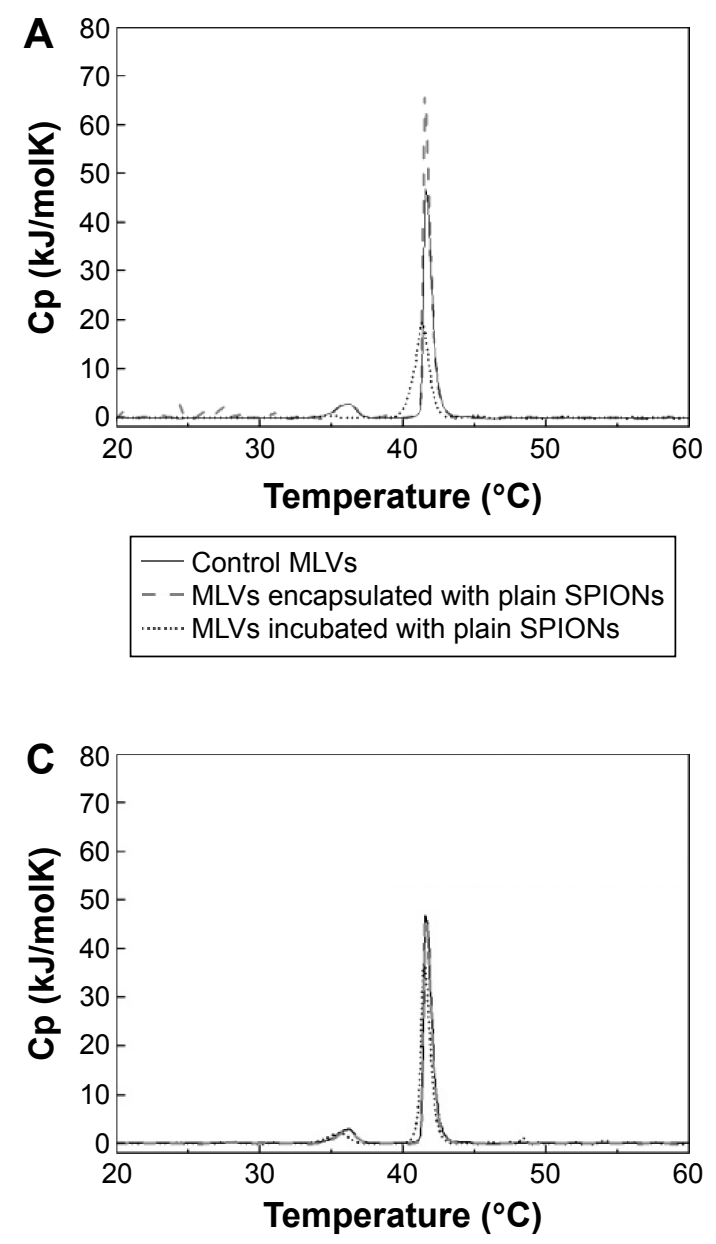

- Control MLVs
- - MLVs encapsulated with carboxyl functionalized
SPIONs
…… MLVs incubated with carboxyl functionalized SPIONs

\section{Effects of SPIONs on the lipid phase transition profile}

Comparison of DSC thermograms of DPPC MLVs encapsulated and incubated with all types of SPIONs suspended in $10 \mathrm{mM}$ HEPES buffer (pH 7.0) is shown in Figure 5A-D. All heating profiles presented with respect to the control sample (pure DPPC MLVs) are in good agreement with the reported characteristic values. ${ }^{38,43-45}$ The thermodynamic profile $\left(T_{m}\right.$ and $\Delta H$ ) of control and DPPC MLVs treated by the incubated and encapsulated SPIONs is reported in Table 3.

Incubation of DPPC MLVs with the plain SPIONs did not induce any alterations in the position of the main transition temperature peak (Table 3 ) with respect to the control, instead
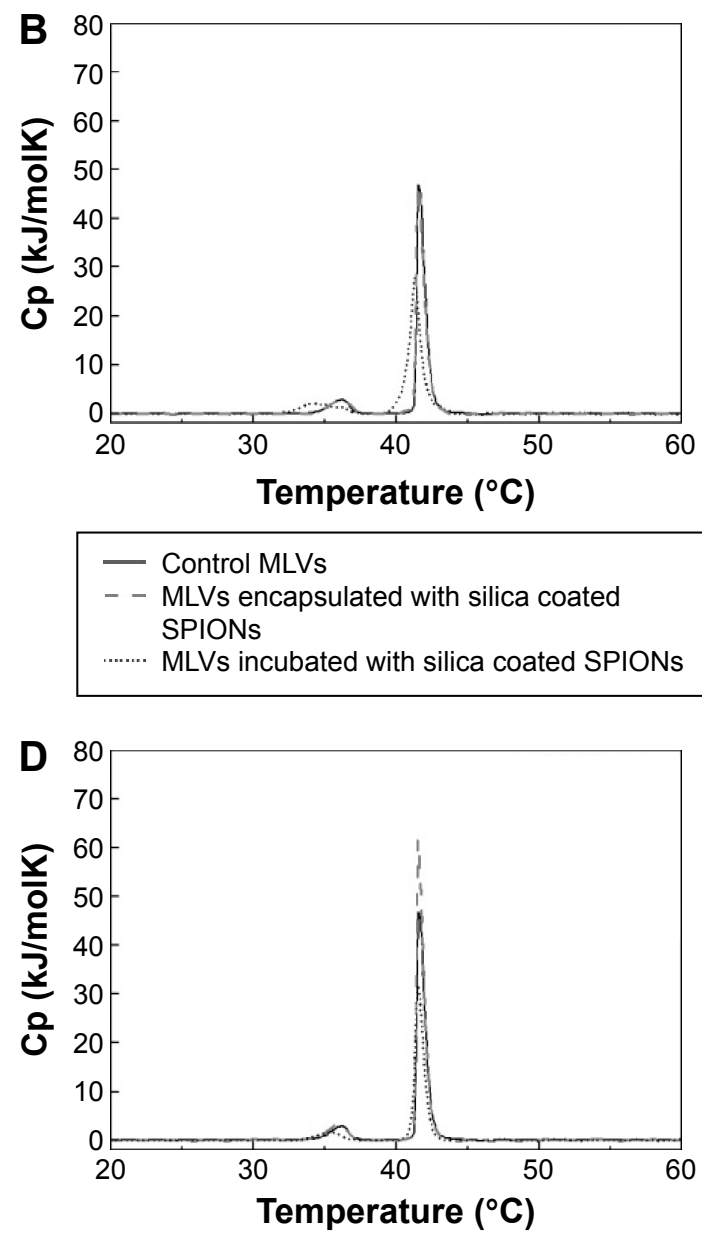

- Control MLVs
- MLVs encapsulated with amino group functionalized SPIONs
SPIONs

Figure 5 DSC thermograms (A-D) showing the interactions DPPC MLVs encapsulated with SPIONs (grey dashed line) and DPPC MLVs incubated with the same type of SPIONs (black dotted line).

Notes: Panels A-D show DSC thermograms for individual SPIONs (encapsulated or incubated) with respect to control SPIONs: (A) plain SPIONs, (B) silica coated SPIONs, (C) SPIONs functionalized with carboxyl groups, and (D) SPIONs functionalized with amino groups.

Abbreviations: MLVs, multilamellar vesicles; SPIONs, superparamagnetic iron oxide nanoparticles; DSC, differential scanning calorimetry; DPPC, I,2-dipalmitoyl-sn-glycero3-phosphocholine; Cp, heat capacity. 
Table 3 Thermodynamic profile of the phase transitions of the DPPC MLVs incubated or encapsulated with the plain and surface modified SPIONs at $\mathrm{pH} 7.0$

\begin{tabular}{|c|c|c|c|c|}
\hline \multirow[t]{2}{*}{ DPPC MLVs } & \multicolumn{2}{|c|}{$\begin{array}{l}\text { DPPC MLVs } \\
\text { with incubated } \\
\text { SPIONs } \\
\end{array}$} & \multicolumn{2}{|c|}{$\begin{array}{l}\text { DPPC MLVs } \\
\text { with encapsulated } \\
\text { SPIONs }\end{array}$} \\
\hline & $\begin{array}{l}\Delta H_{\text {cal }} \\
(\mathrm{kJ} / \mathrm{mol})\end{array}$ & $\begin{array}{l}T_{m} \\
\left({ }^{\circ} \mathrm{C}\right)\end{array}$ & $\begin{array}{l}\Delta H_{c a l} \\
(\mathrm{~kJ} / \mathrm{mol})\end{array}$ & $\begin{array}{l}T_{m} \\
\left({ }^{\circ} \mathrm{C}\right)\end{array}$ \\
\hline Control & $33.7 \pm 3.4$ & $41.6 \pm 0.2$ & $32.4 \pm 3.2$ & $41.5 \pm 0.2$ \\
\hline Plain SPIONs & $40.4 \pm 3.4$ & $41.5 \pm 0.2$ & $26.7 \pm 3.2$ & $41.3 \pm 0.2$ \\
\hline Silica-coated SPIONs & $33.6 \pm 3.4$ & $41.5 \pm 0.2$ & $31.2 \pm 3.2$ & $41.3 \pm 0.2$ \\
\hline $\begin{array}{l}\text { Carboxyl group } \\
\text { functionalized SPIONs }\end{array}$ & $32.0 \pm 3.4$ & $41.6 \pm 0.2$ & $30.2 \pm 3.2$ & $41.5 \pm 0.2$ \\
\hline $\begin{array}{l}\text { Amino group } \\
\text { functionalized SPIONs }\end{array}$ & $38.8 \pm 3.4$ & $41.5 \pm 0.2$ & $25.2 \pm 3.2$ & $41.5 \pm 0.2$ \\
\hline
\end{tabular}

Note: Data are represented as mean \pm standard deviation.

Abbreviations: DPPC, 1,2-dipalmitoyl-sn-glycero-3-phosphocholine; MLVs, multilamellar vesicles; SPIONs, superparamagnetic iron oxide nanoparticles; $\Delta H_{\text {cal }}$ calorimetric enthalpy change of the main phase transition; $T_{m}$, phase transition temperature required to change the membrane lipids from gel to liquid crystalline phase.

the peak height increased (Figure 5A, dashed line), which suggests liposomal membranes incubated with plain SPIONs were subjected to slight stabilization by the adsorbed SPIONs. The heating curve also exhibited slight undulations under $30^{\circ} \mathrm{C}$, probably as a consequence of the sedimentation of SPIONs in the suspension during the measurement (Figure 5A, dashed line). In the case of encapsulated plain SPIONs, the main transition peak was broadened, decreased, and slightly shifted toward lower temperature, while the pretransition peak was almost eliminated (Figure 5A, short dotted line). Similarly, encapsulated silica-coated SPIONs slightly broadened and decreased the main transition peak (Figure 5B, short dotted line), whereas incubation with the same particles (Figure 5B, dashed line) did not show noticeable changes in the shape of DSC thermograms with respect to the control (Figure 5B, straight line). The carboxyl groups functionalized SPIONs did not induce alterations in the shape of DSC thermogram both when incubated and encapsulated (Figure 5C, dashed and short dotted line, respectively). The incubation of DPPC vesicles with amino groups functionalized SPIONs (Figure 5D, dashed line) induced, similarly to the plain SPIONs, an increase in the height of the main transition peak in comparison with the control, but the SPIONs encapsulated inside the vesicles caused a slight decrease in both the main and the pretransition peak; the latter was also moved slightly toward lower temperatures (Figure 5D, dashed line). Therefore, from the above results we conclude that among all types of SPIONs, the SPIONs functionalized with carboxyl groups (correspondingly higher negative $\zeta$-potential values) have the lowest observed effect on the phase transitions of the DPPC MLVs.

\section{Discussion}

We have compared the effects of plain and surface-modified SPIONs on the fluidity and phase transition of the zwitterionic phosphatidylcholine liposomal membranes by two different approaches: 1) SPIONs encapsulated inside the liposomes and 2) SPIONs incubated with the liposomes. The fluorescence anisotropy of POPC SUVs with the encapsulated and incubated SPIONs was measured to assess the fluidity of liposomal membranes. As a complementary method for the assessment of alterations in membrane integrity, calorimetric technique DSC was used to study the effects on the phase transition of DPPC MLVs exposed to SPIONs using the same above-mentioned approaches.

Since the size of the SPIONs exceeds the thickness of the bilayer (primary size of the SPION core was approximately $11 \mathrm{~nm}$ and the silica-coated SPIONs ranges approximately $20 \mathrm{~nm}$ ), we did not expect the incorporation of SPIONs inside the membrane. However, we anticipated that the SPIONs will be encapsulated inside the liposomes. TEM images and the corresponding EDS analysis (Figure 3) have confirmed the presence of silica-coated SPIONs in the liposomes. We assume that during the process of drying the suspension of magnetoliposomes on the grids under vacuum, membrane rupture could have occurred which could most probably be the reason for the invisibility of the multilayer membranes of the MLVs.

\section{Encapsulation efficiency}

It is a prerequisite to have a high EE\% of magnetic NPs in the liposomes to achieve effective targeting, imaging, and therapeutic results. In comparison with the plain SPIONs, silica-coated SPIONs are widely used for in vivo applications due to enhanced stability and biocompatibility. Since they are negatively charged, we have used positively charged SPIONs functionalized with amino groups to compare if the variation in the surface charge of SPIONs has any influence on the $\mathrm{EE} \%$. The $\mathrm{EE} \%$ of magnetoliposomes was calculated using the method described by Frascione et $\mathrm{al}^{4}$ and Skouras et al. ${ }^{42}$ Our results (Table 1) have shown that EE\% of POPC magnetoliposomes loaded with silica-coated SPIONs was comparatively higher than the magnetoliposomes loaded with SPIONs functionalized with amino groups. These results coincide with the results obtained in our previous work ${ }^{26,27}$ where we have demonstrated that due to electrostatic attractions, the negatively charged SPIONs were significantly attracted toward the terminal positive amino group of the POPC liposomal membranes. A lot of reports have also shown that the electrostatic attraction between the NPs and 
the lipid membrane is an important factor to increase both the EE\% of NPs in liposomes and the internalization ratio of NPs in cells..$^{34,46-48}$ The findings of our present study have also shown that $\mathrm{EE} \%$ decreased proportionally with the increase in the concentration of both types of surface-modified SPIONs in magnetoliposomes. The results are in agreement with the results reported earlier, where the authors have demonstrated reduced $\mathrm{EE} \%$ with increased initial iron content. ${ }^{4,42}$

\section{Liposomal membrane fluidity}

Lipid ordering parameter, $S$, is a measure of membrane fluidity of both the biological membranes and the artificial liposomal membranes. We have assessed the lipid ordering parameter of liposomes encapsulated or incubated with the SPIONs. The two common fluorescent probes DPH and TMA-DPH, as shown in Figure 4A-D, were used. The DPH molecules orient loosely between the hydrophobic tails throughout the lipid bilayer and lie parallel to the axis of lipid acyl chains. ${ }^{49}$ TMA-DPH is a cationic derivative of DPH and is located near the phospholipid heads at the membrane-water interface. ${ }^{50}$ Among all the cases depicted in Figure 4A-D, in comparison with the control SUVs, there is a slight increase in the $S$ values of SUVs treated with SPIONs by both the methods, that is, encapsulation and incubation. Figure 4A shows the $S$ values of control SUVs, SUVs encapsulated with plain and surface-modified SPIONs using DPH. The $S$ values decreased steadily for all the samples with a corresponding increase in the temperature, and there was a little difference between the control SUVs and SUVs encapsulated with SPIONs. Since DPH is a hydrophobic probe, the result indicates that all types of the encapsulated SPIONs used in this study caused a slight alteration in the lipid order parameter. Figure 4B shows that the difference in $S$ value between the control SUVs and SUVs encapsulated with SPIONs was almost negligible, which indicates there were not many significant changes in the fluidity level at the membrane lipid heads as detected by TMA-DPH. Figure $4 \mathrm{C}$ shows a considerable difference between the control SUVs and SUVs incubated with SPIONs till $30^{\circ} \mathrm{C}$. As the temperature increased above $30^{\circ} \mathrm{C}$, the difference was almost negligible, which might be due to the sedimentation of larger SPION aggregates. Figure 4D does not show a notable difference in $S$ values between the control SUVs and SUVs incubated with SPIONs and all tested SPIONs using TMA-DPH. On the other hand, the DPH probe (Figure 4C) showed considerable larger differences among the tested SPIONS at lower temperatures. This observed difference in $S$ value between Figure 4C and $\mathrm{D}$ is attributed to two major reasons, the variation in the interaction level of the incubated SPIONs with the membrane lipids at different temperatures, and the nature of the probes. DPH is a very sensitive hydrophobic probe that intercalates itself between the lipid tails of the membrane and responds even to very small alterations in the fluidity level of the membrane interior, whereas TMA-DPH is a surface probe and is less sensitive to temperature. ${ }^{51}$ From the overall results shown in Figure 4A-D, we have observed only slight deviations and no significant changes in $S$ values for both the probes DPH and TMA-DPH, suggesting a general negligible effect of the tested SPIONs on both the hydrophobic lipid tails and the hydrophilic lipid heads. Moreover, among the encapsulated and incubated SPIONs, the amino group functionalized SPIONs showed a slightly higher effect on fluidity than the other types of SPIONs. Due to the little negative surface potential of the prepared liposomes (pure POPC SUVs (control); $-4 \mathrm{mV}$ ), the positively charged amino group modified SPIONs would have attracted more toward the lipid bilayer leading to increased interactions with the membrane lipids, thereby causing significant differences in the lipid ordering. Goertz et $\mathrm{al}^{52}$ have reported that the cationic NPs tend to bend the head group over, expand the lipid bilayer by enhancing the area occupied per head group and cause alterations in lipid ordering and induce phase transition of the bilayer lipids. Though our results have indicated that all types of the studied SPIONs treated by both approaches did not alter the fluidity of the lipid membrane to a significant extent. A similar result was reported by Frascione et al using the polyethylene glycol-coated sterically-stabilized magnetic liposomes treated with the ultrasmall superparamagnetic iron oxide (USPIOs) NPs. ${ }^{4}$ The results have shown that the anisotropy values of POPC liposomes encapsulated with the dextran-coated USPIOs $(<50 \mathrm{~nm})$ were higher than the liposomes incubated with dextran-coated USPIOs. They have explained that this difference was due to the confinement of high concentration of encapsulated NPs in a small volume of water in the hydrophilic region of liposomes, leading to increased interaction of NPs with the inner layer of membrane causing bilayer rigidification and subsequently contributed to increased anisotropy values.

In our previous works, ${ }^{26-28}$ we have compared the influence of electrostatic forces between the positively charged amino group functionalized SPIONs and negatively charged citric acid-coated cobalt ferrite $\left(\mathrm{CoFe}_{2} \mathrm{O}_{4}\right) \mathrm{NPs}(1 \mathrm{mg} / \mathrm{mL}$ suspended in $20 \mathrm{mM}$ HEPES buffer) on zwitterionic 1-stearoyl-2-oleoylsn-glycero-3-phosphocholine (SOPC) liposomes and measured the membrane fluidity. The results have shown that the attractive electrostatic forces between the negatively charged 
$\mathrm{CoFe}_{2} \mathrm{O}_{4}$ NPs and the terminal positive amino group of the SOPC lipid enhanced the interaction of NPs with the membrane lipids causing slight alterations in the fluidity level. On the other hand, due to electrostatic repulsion between the positively charged SPIONs and the terminal positive amino group of the SOPC lipid, the approach and interaction of the SPIONs with the lipid membrane was less leading to no noticeable differences in the fluidity level.

To take our work to the next level, we have compared the effect of encapsulated and incubated SPIONs with different surface charges on the fluidity of POPC zwitterionic liposomes. Since lower concentrations of NPs are commonly used for clinical applications to avoid unwanted side effects, we have used low concentrations of SPIONs $(0.05 \mathrm{mg} / \mathrm{mL}$ suspended in $10 \mathrm{mM}$ HEPES buffer) in this work, hoping that these results can be considered for our future works on cell membrane fluidity using these SPIONs. In the present study, the fluidity measurements that were not significantly affected by the encapsulated or incubated SPIONs were in line with our expectations. Our results have further confirmed the previous reports ${ }^{16,46,53-55}$ that there was no major difference in the membrane fluidity level due to the surface-modified SPIONs and hence they are suitable to be used for in vivo applications.

\section{Phase transition of liposomal membrane}

The phase transition temperature denotes the temperature $\left(T_{m}\right)$ at which an alteration in the phase of membrane lipids occurs, from the ordered gel phase to the disordered liquid crystalline phase. In the gel phase, the hydrocarbon chains are completely extended and tightly packed, whereas they are loosely packed and more fluid in the liquid crystalline phase. ${ }^{56}$ When the length of the hydrocarbon chain increases, the strength of the van der Waals interactions also increases. To disrupt the packing order of the lipid chains, a greater amount of energy is required and thus the $T_{m}$ increases. ${ }^{51,57,58}$ We have observed no major alterations in the thermal stability of DPPC vesicles, since the $T_{m}$ of fully hydrated DPPC MLVs detected at $41^{\circ} \mathrm{C}$ did not change neither with the encapsulated nor with the incubated SPIONs. We have observed some alterations in the DPPC peak shapes in the interaction of encapsulated SPIONs with DPPC. The encapsulation of plain SPIONs and SPIONs functionalized with amino groups exhibited a higher main transition peak with respect to the control (ie, pure DPPC MLVs in $10 \mathrm{mM}$ HEPES buffer at $\mathrm{pH}$ 7.0), which can be explained as a slight stabilization of the membrane upon contact between SPIONs and the charged groups of the membrane. The shape of the main transition peak (higher, narrower) suggests that the phospholipids possess higher lipid order after incubation with the SPIONs functionalized by amino group which is in line with the fluidity results showing the plain SPIONs (Figure 4A; empty squares) and broadened main transition peaks to a small extent (Figure 5A and B, respectively); the pre-transition peak was almost eliminated in MLVs incubated with plain SPIONs (Figure 5A; dashed line). Generally, the incubation of DPPC with SPIONs has a noticeable effect on DSC thermograms: lowered, broadened, and moved toward lower temperatures with plain, silica-coated, and functionalized with amino group (Figure 5A, B, and D, respectively), but also slightly lowered with incubated SPIONs functionalized with carboxyl group (Figure 5C). On the other hand, the fluidity results for the encapsulated amino-functionalized SPIONs (Figure 4) are not in line with the observation. The DPPC membranes were observed to be the most intact and unaffected by the SPIONs functionalized with carboxyl groups using both the encapsulated and incubated SPIONs (Figure 5C). In line with the measured $\zeta$-potential values in the HEPES buffer at pH 7.0 (Table 1), the silica-coated SPIONs, possessing the largest negative surface charge $(-24.3 \mathrm{mV})$, caused a notable effect on the phase transition profile of DPPC MLVs, stronger than the plain SPIONs $(-5.0 \mathrm{mV})$ and SPIONs functionalized amino groups $(+4.1 \mathrm{mV})$, and the SPIONs functionalized with carboxyl group $(-18.4 \mathrm{mV})$; the latter were observed to have the lowest effect on the phase transition of DPPC. However, based on our observations, we cannot make very straightforward conclusions as, for instance, demonstrated by Chen et al. ${ }^{59}$ They have reported on the embedding of SPIONs within DPPC lipid bilayers on the basis of both the main transition peak broadening and the shift of main transition temperature peak to higher temperatures. Moreover, changes in the thermodynamic state of lipid membranes can also be provoked due to the NP dissolution in aqueous dispersions. ${ }^{37}$ The chemical characterization data and magnetic measurements of the SPIONs in our present study showed no or very subtle particle dissolution (data not shown), which suggests that the observed slight alterations in the thermodynamic profile are due to mechanical rather than chemical mechanisms. ${ }^{37}$ The $\zeta$-potential value of DPPC MLVs prepared under the same experimental conditions was reported to be $-7 \pm 20 \mathrm{mV} .{ }^{60}$ Due to the zwitterionic form of the liposome membranes, we cannot explain the effect of SPIONs on the thermal profile of membrane lipids solely on the basis of electrostatic interactions. Based on the results, we conclude that the interactions of surface-modified SPIONs with the membrane depend on various factors such as surface 
charge, size of agglomerates formed in the suspension, and the number of particles interacting with the phospholipid bilayer. ${ }^{61}$

\section{Conclusion}

The prospective of our work is to contribute to a better understanding of the interactions of the plain and surface-modified SPIONs treated by both approaches, that is, encapsulation and incubation with a zwitterionic lipid bilayer, which is important for optimizing the characteristics of SPIONs to make them ideal for diverse biomedical applications. Based on our results, we conclude that these SPIONs at relatively low concentration (eg, $0.05 \mathrm{mg} \mathrm{Fe}_{2} \mathrm{O}_{3} / \mathrm{mL}$ ) did not have a significant effect on the lipid ordering, membrane fluidity, and phase transition behavior of phosphatidylcholine liposomes. Since the membrane properties are not altered, the results indicate that irrespective of the method of exposure (encapsulation or incubation), the SPIONs did not cause any rupture or damage to the membrane under the given experimental conditions, otherwise it would have led to significant alterations in the studied membrane properties. It is also worthy to specify that it is not just the concentration of SPIONs that affects the biophysical properties of the membrane but a combination of a number of factors including the number of SPIONs interacting with the liposomes, as well as their size, shape, and surface charge have to be taken into account while considering these NPs for biophysical and in vivo investigations. The present study is an upgrade of our previously published results and suggests the suitability of these SPIONs for encapsulation in phosphatidylcholine liposomes for clinical purpose. Hence, our further studies are underway taking into account all these factors including the pharmacological and toxicological studies of SPIONs using animal models to gain extensive knowledge about the suitability of these SPIONs for theranostic applications.

\section{Acknowledgments}

This work was supported by the Slovene Human Resources Development and Scholarship Fund (Grant no 110139/2012-6). The investigation was also supported by the Ministry of Education, Science, Culture and Sport of Republic of Slovenia under a grant "Innovative scheme of co-funding doctoral studies promoting co-operation with the economy and solving of contemporary social challenges" (Grant no 160-21). This project has also received funding from the Slovenian Research Agency (ARRS) under grant agreement no J1-4109, P4-0121 and P3-0108. We acknowledge the use of equipment in the Center of Excellence on
Nanoscience and Nanotechnology-Nanocenter. The authors would like to thank Dr Janez Valant, Dr Ajda Ota, and Linda Štrus for their technical assistance.

\section{Disclosure}

The authors report no conflicts of interest in this work.

\section{References}

1. Bealle G, Di Corato R, Kolosnjaj-Tabi J, et al. Ultra magnetic liposomes for MR imaging, targeting, and hyperthermia. Langmuir. 2012;28(32): 11843-11851.

2. Pradhan P, Banerjee R, Bahadur D, Koch C, Mykhaylyk O, Plank C. Targeted magnetic liposomes loaded with doxorubicin. Methods Mol Biol. 2010;605:279-293.

3. Huang J, Wang LY, Lin R, et al. Casein-coated iron oxide nanoparticles for high MRI contrast enhancement and efficient cell targeting. ACS Appl Mater Inter. 2013;5(11):4632-4639.

4. Frascione D, Diwoky C, Almer G, et al. Ultrasmall superparamagnetic iron oxide (USPIO)-based liposomes as magnetic resonance imaging probes. Int J Nanomed. 2012;7:2349-2359.

5. Ling DS, Hyeon T. Chemical design of biocompatible iron oxide nanoparticles for medical applications. Small. 2013;9(9-10):1450-1466.

6. Mikhaylov G, Mikac U, Magaeva AA, et al. Ferri-liposomes as an MRI-visible drug-delivery system for targeting tumours and their microenvironment. Nat Nanotechnol. 2011;6(9):594-602.

7. Zhang XQ, Xu X, Bertrand N, Pridgen E, Swami A, Farokhzad OC. Interactions of nanomaterials and biological systems: implications to personalized nanomedicine. Adv Drug Deliver Rev. 2012;64(13):1363-1384.

8. Laurent S, Dutz S, Hafeli UO, Mahmoudi M. Magnetic fluid hyperthermia: focus on superparamagnetic iron oxide nanoparticles. Adv Colloid Interfac. 2011;166(1-2):8-23.

9. Kolhatkar AG, Jamison AC, Litvinov D, Willson RC, Lee TR. Tuning the magnetic properties of nanoparticles. Int J Mol Sci. 2013;14(8): 15977-16009.

10. Santhosh PB, Ulrih NP. Multifunctional superparamagnetic iron oxide nanoparticles: promising tools in cancer theranostics. Cancer Lett. 2013; 336(1):8-17.

11. Barua S, Mitragotri S. Challenges associated with penetration of nanoparticles across cell and tissue barriers: a review of current status and future prospects. Nano Today. 2014;9(2):223-243.

12. Wang B, Zhang LF, Bae SC, Granick S. Nanoparticle-induced surface reconstruction of phospholipid membranes. Proc Natl Acad Sci US A. 2008;105(47):18171-18175.

13. Bhandary S, Sultana P, Basu R, Das S, Nandy P. A Study on the modulation of the phase behavior of lipid aggregates-effect of some metal nanoparticles. Adv Sci Eng Med. 2011;3(3):213-218.

14. Schulz M, Olubummo A, Binder WH. Beyond the lipid-bilayer: interaction of polymers and nanoparticles with membranes. Soft Matter. 2012; 8(18):4849-4864.

15. Yu M, Huang SH, Yu KJ, Clyne AM. Dextran and polymer polyethylene glycol (PEG) coating reduce both 5 and $30 \mathrm{~nm}$ iron oxide nanoparticle cytotoxicity in 2D and 3D cell culture. Int J Mol Sci. 2012; 13(5):5554-5570.

16. Carvalho A, Martins MBF, Corvo ML, Feio G. Enhanced contrast efficiency in MRI by PEGylated magnetoliposomes loaded with PEGylated SPION: Effect of SPION coating and micro-environment. Mat Sci Eng C Mater Biol Appl. 2014;43:521-526.

17. Markides H, Kehoe O, Morris RH, El Haj AJ. Whole body tracking of superparamagnetic iron oxide nanoparticle-labelled cells - a rheumatoid arthritis mouse model. Stem Cell Res Ther. 2013;4(5):126.

18. Verma VK, Kamaraju SR, Kancherla R, et al. Fluorescent magnetic iron oxide nanoparticles for cardiac precursor cell selection from stromal vascular fraction and optimization for magnetic resonance imaging. Int J Nanomed. 2015;10:711-726. 
19. Franke K, Kettering M, Lange K, Kaiser WA, Hilger I. The exposure of cancer cells to hyperthermia, iron oxide nanoparticles, and mitomycin $\mathrm{C}$ influences membrane multidrug resistance protein expression levels. Int J Nanomed. 2013;8:351-363.

20. Gonzales M, Krishnan KM. Synthesis of magnetoliposomes with monodisperse iron oxide nanocrystal cores for hyperthermia. J Magn Magn Mater. 2005;293(1):265-270.

21. Martins MB, Corvo ML, Marcelino P, Marinho HS, Feio G, Carvalho A. New long circulating magnetoliposomes as contrast agents for detection of ischemia-reperfusion injuries by MRI. Nanomedicine 2014;10(1):207-214.

22. Thomas R, Park IK, Jeong YY. Magnetic iron oxide nanoparticles for multimodal imaging and therapy of cancer. Int J Mol Sci. 2013; 14(8):15910-15930.

23. Peetla C, Labhasetwar V. Biophysical characterization of nanoparticleendothelial model cell membrane interactions. Mol Pharm. 2008;5(3): $418-429$

24. RoiterY, Ornatska M, Rammohan AR, Balakrishnan J,Heine DR, Minko S Interaction of nanoparticles with lipid membrane. Nano Lett. 2008; 8(3):941-944.

25. Park SH, Oh SG, Mun JY, Han SS. Effects of silver nanoparticles on the fluidity of bilayer in phospholipid liposome. Colloid Surface B. 2005; 44(2-3):117-122.

26. Velikonja A, Santhosh PB, Gongadze E, et al. Interaction between dipolar lipid headgroups and charged nanoparticles mediated by water dipoles and ions. Int J Mol Sci. 2013;14(8):15312-15329.

27. Santhosh PB, Velikonja A, Perutkova S, et al. Influence of nanoparticlemembrane electrostatic interactions on membrane fluidity and bending elasticity. Chem Phys Lipids. 2014;178:52-62.

28. Santhosh PB, Penic S, Genova J, Iglic A, Kralj-Iglic V, Ulrih NP A study on the interaction of nanoparticles with lipid membranes and their influence on membrane fluidity. J Phys Conf Ser. 2012;398:012034.

29. Campelj S, Makovec D, Drofenik M. Preparation and properties of waterbased magnetic fluids. J Phys-Condens Mat. 2008;20(20):204101

30. Kralj S, Makovec D, Campelj S, Drofenik M. Producing ultra-thin silica coatings on iron-oxide nanoparticles to improve their surface reactivity. J Magn Magn Mater. 2010;322(13):1847-1853.

31. Kralj S, Drofenik M, Makovec D. Controlled surface functionalization of silica-coated magnetic nanoparticles with terminal amino and carboxyl groups. J Nanopart Res. 2011;13(7):2829-2841.

32. Kralj S, Makovec D. The chemically directed assembly of nanoparticle clusters from superparamagnetic iron-oxide nanoparticles. RSC Adv. 2014;4(25):13167-13171.

33. Lasic D. Liposomes: From Physics to Applications. Amsterdam: New York: Elsevier; 1993.

34. Sabate R, Barnadas-Rodriguez R, Callejas-Fernandez J, HidalgoAlvarez R, Estelrich J. Preparation and characterization of extruded magnetoliposomes. Int J Pharmaceut. 2008;347(1-2):156-162.

35. Pottel H, Vandermeer W, Herreman W. Correlation between the order parameter and the steady-state fluorescence anisotropy of 1,6-Diphenyl1,3,5-Hexatriene and an evaluation of membrane fluidity. Biochim Biophys Acta. 1983;730(2):181-186.

36. Seelig J. Thermodynamics of lipid-peptide interactions. Biochim Biophys Acta. 2004;1666(1-2):40-50.

37. Westerhausen C, Strobl FG, Herrmann R, et al. Chemical and mechanical impact of silica nanoparticles on the phase transition behavior of phospholipid membranes in theory and experiment. Biophys J. 2012;102(5): 1032-1038.

38. Ota A, Abramovic H, Abram V, Ulrih NP. Interactions of p-coumaric caffeic and ferulic acids and their styrenes with model lipid membranes. Food Chem. 2011;125(4):1256-1261.

39. Philosof-Mazor L, Dakwar GR, Popov M, et al. Bolaamphiphilic vesicles encapsulating iron oxide nanoparticles: new vehicles for magnetically targeted drug delivery. Int J Pharm. 2013;450(1-2):241-249.

40. Elersic K, Pavlic JI, Iglic A, Vesel A, Mozetic M. Electric-field controlled liposome formation with embedded superparamagnetic iron oxide nanoparticles. Chem Phys Lipids. 2012;165(1):120-124.
41. Faure C, Meyre ME, Trepout S, Lambert O, Lebraud E. Magnetic multilamellar liposomes produced by in situ synthesis of iron oxide nanoparticles: "magnetonions". J Phys Chem B. 2009;113(25):8552-8559.

42. Skouras A, Mourtas S, Markoutsa E, et al. Magnetoliposomes with high USPIO entrapping efficiency, stability and magnetic properties. Nanomedicine. 2011;7(5):572-579.

43. Poklar N, Fritz J, Macek P, Vesnaver G, Chalikian TV. Interaction of the pore-forming protein equinatoxin II with model lipid membranes: a calorimetric and spectroscopic study. Biochemistry. 1999;38(45):14999-15008.

44. Lichtenberg D, Menashe M, Donaldson S, Biltonen RL. Thermodynamic characterization of the pretrasition of unilamellar dipalmitoylphosphatidylcholine vesicles. Lipids. 1984;19(6):395-400.

45. Boggs JM. Lipid intermolecular hydrogen-bonding - influence on structural organization and membrane-function. Biochim Biophys Acta. 1987;906(3):353-404.

46. Santhosh PB, Kiryakova SI, Genova JL, Ulrih NP. Influence of iron oxide nanoparticles on bending elasticity and bilayer fluidity of phosphotidylcholine liposomal membranes. Colloid Surface A. 2014;460:248-253.

47. Zhu XM, Wang YXJ, Leung KCF, et al. Enhanced cellular uptake of aminosilane-coated superparamagnetic iron oxide nanoparticles in mammalian cell lines. Int J Nanomed. 2012;7:953-964.

48. Schweiger C, Hartmann R, Zhang F, Parak WJ, Kissel TH, Rivera Gil P. Quantification of the internalization patterns of superparamagnetic iron oxide nanoparticles with opposite charge. J Nanobiotechnol. 2012;10:28.

49. Hurjui I, Neamtu A, Dorohoi DO. The interaction of fluorescent DPH probes with unsaturated phospholipid membranes: A molecular dynamics study. J Mol Struct. 2013;1044:134-139.

50. Kuhry JG, Fonteneau P, Duportail G, Maechling C, Laustriat G. TmaDph - a Suitable Fluorescence polarization probe for specific plasmamembrane fluidity studies in intact living cells. Cell Biophys. 1983;5(2): 129-140.

51. Gmajner D, Grabnar PA, Znidaric MT, Strus J, Sentjurc M, Ulrih NP. Structural characterization of liposomes made of diether archaeal lipids and dipalmitoyl-L-alpha-phosphatidylcholine. Biophys Chem. 2011; 158(2-3):150-156.

52. Goertz MP, Goyal N, Bunker BC, Montano GA. Substrate effects on interactions of lipid bilayer assemblies with bound nanoparticles. J Colloid Interf Sci. 2011;358(2):635-638.

53. Lundquist CM, Loo C, Meraz IM, Cerda JD, Liu X, Serda RE. Characterization of free and porous silicon-encapsulated superparamagnetic iron oxide nanoparticles as platforms for the development of theranostic vaccines. Med Sci. 2014;2(1):51-69.

54. Malvindi MA, De Matteis V, Galeone A, et al. Toxicity assessment of silica coated iron oxide nanoparticles and biocompatibility improvement by surface engineering. PLoS One. 2014;9(1):e85835.

55. Wahajuddin, Arora S. Superparamagnetic iron oxide nanoparticles: magnetic nanoplatforms as drug carriers. Int J Nanomed. 2012;7: 3445-3471.

56. Leonenko ZV, Finot E, Ma H, Dahms TES, Cramb DT. Investigation of temperature-induced phase transitions in DOPC and DPPC phospholipid bilayers using temperature-controlled scanning force microscopy. Biophys J. 2004;86(6):3783-3793.

57. Kastantin M, Ananthanarayanan B, Karmali P, Ruoslahti E, Tirrell M. Effect of the lipid chain melting transition on the stability of DSPE-PEG (2000) Micelles. Langmuir. 2009;25(13):7279-7286.

58. Wrobel D, Klys A, Ionov M, et al. Cationic carbosilane dendrimers-lipid membrane interactions. Chem Phys Lipids. 2012;165(4):401-407.

59. Chen YJ, Bose A, Bothun GD. Controlled release from Bilayer-decorated magnetoliposomes via electromagnetic heating. ACS Nano. 2010;4(6): 3215-3221.

60. Gmajner D, Ota A, Sentjurc M, Ulrih NP. Stability of diether C-25, C-25 liposomes from the hyperthermophilic archaeon Aeropyrum pernix K1. Chem Phys Lipids. 2011;164(3):236-245.

61. Schladt TD, Schneider K, Schild H, Tremel W. Synthesis and biofunctionalization of magnetic nanoparticles for medical diagnosis and treatment. Dalton T. 2011;40(24):6315-6343. 


\section{Publish your work in this journal}

The International Journal of Nanomedicine is an international, peerreviewed journal focusing on the application of nanotechnology in diagnostics, therapeutics, and drug delivery systems throughout the biomedical field. This journal is indexed on PubMed Central, MedLine, CAS, SciSearch $\AA$, Current Contents $\AA /$ Clinical Medicine,

Journal Citation Reports/Science Edition, EMBase, Scopus and the Elsevier Bibliographic databases. The manuscript management system is completely online and includes a very quick and fair peer-review system, which is all easy to use. Visit http://www.dovepress.com/ testimonials.php to read real quotes from published authors.

Submit your manuscript here: http://www.dovepress.com/international-journal-of-nanomedicine-journal 